\title{
TUTORÍA VIRTUAL PARA PROFESIONALES DE ENFERMERÍA NOVELES
}

(A VIRTUAL BASED TUTORIAL FOR NEW NURSING PROFESSIONALS)

\author{
Mar Lleixà Fortuño \\ Mercè Gisbert-Cervera \\ Luis Marqués-Molías \\ Cinta Espuny Vidal \\ Juan González Martínez \\ Nuria Albacar Riobóo \\ Universitat Rovira i Virgili, Campus Terres de l'Ebre (España)
}

\section{RESUMEN}

Esta investigación expone el proceso de implantación de un programa de tutoría virtual para los profesionales noveles de enfermería durante su inserción laboral, valorado positivamente por los participantes en términos de motivación, ayuda y acompañamiento a la inserción, así como por lo que respecta a su duración, a la información y el apoyo ofrecidos. Tras esta experiencia piloto, se destaca la necesidad e importancia de que el programa continúe para poder responder a la necesidad de apoyo y soporte emocional detectado; asimismo, la experiencia pretende servir como punto de partida para institucionalizar un programa virtual de inserción laboral para profesionales de enfermería noveles en el contexto del sistema público de salud.

Palabras clave: profesional de enfermería novel, tecnologías de la información y comunicación (TIC), tutoría on-line, mentoring, soporte emocional, tecnología educativa.

\section{ABSTRACT}

This research study presents the implementation of a virtual tutorial system for new Nursing professionals during their work placements, which is positively evaluated by the participants in terms of motivation, academic support, etc.

Following this pilot experience, one can highlight the need and importance of continuing with the program so that it continues to respond to their needs in terms of student help and support. Similarly, the initiative aims to serve as a starting point for institutionalizing a virtual 
program of work placements for new nursing professionals, i.e. within the context of public health professionals.

Keywords: new nursing professionals, ICTs, on-line tutoring, mentoring, emotional support, educational technology.

Los programas de inserción en los primeros años de desarrollo profesional están considerados como uno de los factores claves para conseguir una incorporación exitosa a la institución (Ezeiza, 2007). De hecho, durante la pasada década, la adopción masiva de las Tecnologías de la Información y de la Comunicación (TIC) ha abierto nuevas oportunidades para minimizar distancias entre las personas, de las que los programas de tutoría también se han beneficiado (Rísquez, 2006; Sedeño, 2006; Ezeiza, 2007).

Como sabemos, la tutoría se refiere al conjunto de acciones propias de la figura que ejerce protección y tutela de otra persona menor, entre cuyos cometidos principales encontramos el fomento del desarrollo del estudio independiente, esto es, se trata de un orientador del aprendizaje que facilita las situaciones de aprendizaje y ayuda a resolver las diferentes dificultades (García Aretio, 2001). Y, por lo que a nosotros nos atañe, cuando esta tutoría se desarrolla y gestiona mediante un entorno virtual de aprendizaje (EVA), hablamos de tutoría electrónica (Montserrat, Gisbert e Isus, 2007; Montserrat, 2010). En España, una de las primeras iniciativas informatizadas de orientación, centrada en el ámbito académico-universitario fueron los programas Magallanes (2000) y Simus (2001), ambos desarrollados por un grupo de investigadores de la Universidad de Sevilla (Rodríguez y Valverde, 2003).

En este contexto, la revisión bibliográfica realizada sobre la existencia, el diseño y/o el funcionamiento de programas virtuales de tutoría en enfermería aporta una visión positiva y alentadora, ya que hay diferentes experiencias tanto en el ámbito docente como asistencial sobre la utilización de comunidades virtuales para implantar programas de tutorización (Pérez Juarez, Verdú y otros, 1998; Pallof y Pratt, 1999; Marabotto y Grau, 1999; Scott, 2005; Vizcaya, Pérez et al., 2006; Effken, Boyle e Isenberg, 2008; Lleixá, 2008, 2010a, 2010b). Algunas de estas experiencias coinciden en señalar la potenciación de insertar las ventajas de las TIC al proceso tutorial y añadir flexibilidad al proceso, lo cual en muchos casos ha permitido alargar el tiempo de tutorización y, por tanto, de acompañamiento a los profesionales noveles optimizando los recursos. En todos los casos, los programas de e-tutoría han resultado positivamente evaluados por los participantes implicados, hecho que anima a diseñar e implantar un sistema de e-tutoría destinado a profesionales noveles enfermeros. 
En nuestra experiencia, partimos de la necesidad de apoyo al proceso de inserción laboral de los profesionales de enfermería. Para dar respuesta a esta problemática se decidió diseñar, desarrollar y poner en marcha un programa de tutorización virtual (en adelante, VIRTICS) como sistema de acompañamiento al profesional novel en el proceso de inserción y adaptación al mundo profesional. Este programa de intervención tiene como objetivos:

- Favorecer el fomento de la seguridad personal.

- Disminuir el grado de estrés percibido que provoca la inserción laboral.

- Aumentar el nivel competencial.

- Optimizar los recursos existentes de apoyo a la inserción laboral de los profesionales debutantes.

En el momento de la planificación de VIRTICS, se apostó por un programa de tutoría virtual, como complemento en algunos casos de un programa de tutorización presencial ya existente que daba soporte los primeros días de inserción, el programa propuesto puede ser un modelo óptimo para cubrir la necesidad de apoyo y acompañamiento de los profesionales de enfermería noveles, ya que ofrece todo un conjunto de ventajas, facilita la interacción y la comunicación entre los diferentes participantes alargando la tutorización en el tiempo. Recordemos que el Institut Català de la Salut (ICS), contexto del programa, es una institución que ha apostado muy fuerte por las TIC, y como muestra de ello las tiene muy integradas en el día a día y en su modelo de gestión. Así pues, se aprovecharon todas las posibilidades y ventajas que estas aportaban al proceso tutorial, lo cual facilitó la consecución de los objetivos de la tutoría de una forma cómoda y flexible para todas las personas participantes.

El programa pretendía ser un proyecto innovador que apostó e incorporó las TIC al proceso tutorial, por medio de un sistema de fácil uso y resolutivo donde el profesional debutante tenga un cibertutor que le pueda ayudar y dar el apoyo y el soporte emocional que precisa. Por último, antes de finalizar esta introducción, debemos puntualizar que este programa forma parte de la investigación en que se sustentaba la tesis doctoral de una de las autoras (Lleixà, 2008). 


\section{MATERIAL Y MÉTODOS}

La experiencia se llevó a cabo en el contexto de los profesionales noveles de enfermería incorporados en el Institut Català de la Salut (ICS) del ámbito hospitalario de la Región Sanitaria de las «Terres de l'Ebre». El motivo principal de la limitación fue la proximidad geográfica, circunstancia que garantiza la accesibilidad y el conocimiento por parte de los autores de las instituciones, gestores, profesionales de enfermería, tutores implicados en el estudio... La posibilidad de ampliar el estudio a otras regiones sanitarias y/o proveedores se tuvo en cuenta desde un principio, pero para una segunda fase de ampliación en la cual se está trabajando actualmente.

Al presentar este proyecto a los gestores del ICS, el principal proveedor de servicios de salud del CatSalut, sus gestores lo valoraron positivamente y nos animaron a iniciarlo manifestando en todo momento el interés por el proyecto. Sin duda, esto fue decisivo para centrarlo en nuestra zona sanitaria, pero como ya hemos dicho con la idea de ampliar el contexto en un futuro próximo. Otro aspecto importante para decidir el contexto de la investigación fue que las dos instituciones implicadas (URVICS) ya tenían firmado un convenio de colaboración, circunstancia que facilitó todo el proceso y que puede favorecer la implicación de todos los participantes implicados.

La presente investigación es, por lo que respecta a su tipología, un estudio de caso, ya que la experiencia se llevó a cabo con los cuatro profesionales de enfermería noveles que se incorporaron durante los primeros meses de inserción laboral en el Hospital de Tortosa Verge de la Cinta (HTVC). La duración prevista del programa fue de tres meses, desde julio hasta septiembre. La tutora fue una enfermera asistencial experta del mismo hospital con experiencia docente; y la coordinadora del espacio coincide con una de las autoras del artículo, quien realizó tareas de apoyo en todo momento hacia la tutora y compartió las tareas de moderadora manteniendo la presencia e interacción constante con las diferentes participantes.

\section{Diseño de VIRTICS}

Se parte del análisis del entorno virtual de tutoría (EVT) de la Universidad Rovira i Virgili (URV) integrado en la herramienta de campus virtual de la URV, la plataforma Moodle. De este trabajo ha derivado en Moodle Tutorías, que dispone de las funcionalidades propias de Moodle y de funcionalidades incorporadas para dar respuesta al desarrollo de las e-tutorías. Una vez revisado el EVT se vio que se adaptaba a las necesidades concretas de VIRTICS, partiendo del hecho que es 
un sistema de tutorización para profesionales de enfermería noveles (Montserrat, Gisbert et al., 2005, 2007; Gisbert, Montserrat y Holgado, 2008).

VIRTICS tenía un espacio propio dentro del entorno virtual de aprendizaje Moodle de la URV de Tarragona. El paso previo al diseño del espacio consistió en estructurar y definir el área de Moodle de VIRTICS, eso se llevó a cabo a partir de la revisión y adaptación del EVT de la URV, del plan de acción tutorial (PAT) de la URV y de otra documentación referente con el tema; se realizaron varias reuniones con los gestores de los centros y posibles tutores, hasta llegar a decidir el espacio provisional VIRTICS. Este se presentó a las gestoras para hacer las mejoras consideradas oportunas hasta llegar al diseño definitivo.

Se diseñó un programa de e-tutoría, «VIRTICS», para implantarlo en el verano 2008, a los profesionales de enfermería noveles que se incorporaran al HTVC, como sistema de acompañamiento y apoyo a la incorporación laboral; se reflexionó sobre las potencialidades de las actividades diseñadas en los entornos virtuales, donde la colaboración debía tener un papel prioritario; se trata, en todo caso, de lograr un contexto que enfatizara las interacciones donde los miembros participan autónomamente en un proceso de aprendizaje, mientras resuelven un problema como grupo (Pavón y Casanova, 2007).

Los contenidos de referencia son de orientación profesional, toma de decisiones y resolución de conflictos.

A continuación se describen las diferentes herramientas de comunicación incluidas:

- Foros de discusión: herramienta asíncrona, se activaron diferentes foros estructurados según temas, el nombre del foro iba acompañado de una pequeña explicación del tema y objetivo. Todos los mensajes de los foros llegaban por correo electrónico, ya que la coordinadora suscribió a todos para que la plataforma hiciera llegar estos correos. Se abrieron los siguientes foros:

- Bienvenida y presentación.

- Noticias y anuncios.

- Dudas.

- Acuerdos grupo de discusión.

o Autoevaluación competencias enfermeras.

o Propuesta de chat. 
- Información sobre formación.

- Propuestas de mejora.

o Despedida.

- Correo electrónico: herramienta asíncrona, se utilizaba fundamentalmente para recuperar la atención de aquellos participantes que hacía días que estaban ausentes y para establecer la comunicación entre las tutoras y coordinadora.

- Chat: herramienta síncrona, se realizó una actividad programada, para evaluar la experiencia de la inserción laboral, la inclusión dentro del programa de tutoría virtual...

Se incluyeron diferentes recursos de apoyo dentro del EVT como material que podía ser consultado por los profesionales noveles.

También se incluyeron dentro del bloque específico de tutorías diferentes instrumentos, en formato web, para evaluar el proceso de inserción laboral, y también, el programa de tutorización VIRTICS:

- Ficha web diagnóstica.

- Ficha web final.

- Cuestionario web de evaluación VIRTICS tutorizados.

- Cuestionario web de evaluación VIRTICS tutoras.

\section{Tutories}

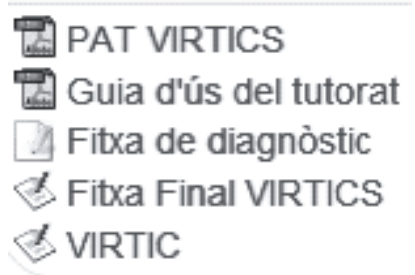

Imagen 1. Funcionalidades específicas de la tutoría

- Metodología: se centra en la gestión de las herramientas de comunicación, tanto asíncronas (foro, correo electrónico), como síncronas (chat), que permite la plataforma Moodle, se parte de un plan de trabajo establecido y orientativo de 
funcionamiento que es facilitado a la tutora dentro de la guía de uso elaborada; en ella se detallan ejemplos de mensajes. Este plan pretende ser un ejemplo que dependerá siempre de las demandas concretas que realicen los tutorados, pero que señala actividades mínimas a desarrollar, definiendo los momentos y objetivos para cada actividad (Ezeiza, 2007).

A continuación se presenta un cuadro resumen del plan de trabajo diseñado:

\begin{tabular}{|c|c|c|c|c|}
\hline FASE & CRONOGRAMA & MODALIDAD & OBJETIVOS & COMO \\
\hline I & $\begin{array}{l}\text { Previo tutoría } \\
\text { final junio }\end{array}$ & Presencial & $\begin{array}{l}\text { Conocer a los alumnos. } \\
\text { Presentar la tutoría. } \\
\text { Facilitar la comunicación. }\end{array}$ & $\begin{array}{l}\text { Curso nueva } \\
\text { incorporación }\end{array}$ \\
\hline II & $\begin{array}{l}\text { Inicio contrato } \\
\text { julio }\end{array}$ & Virtual grupo & $\begin{array}{l}\text { Conocer a los alumnos. } \\
\text { Presentar la tutoría. } \\
\text { Facilitar la comunicación. } \\
\text { Valorar nivel competencias } \\
\text { profesionales. } \\
\text { Valorar el nivel de estrés } \\
\text { percibido. }\end{array}$ & $\begin{array}{l}\text { Foro discusión: } \\
\text { (bienvenida) } \\
\text { Correo } \\
\text { electrónico: } \\
\text { (bienvenida) } \\
\text { Foro: } \\
\text { (recordar ficha } \\
\text { diagnóstica) } \\
\text { Grupo discusión: } \\
\text { (análisis } \\
\text { experiencia) }\end{array}$ \\
\hline III & Julio-agosto & Virtual grupo & $\begin{array}{l}\text { Facilitar la comunicación. } \\
\text { Detectar posibles } \\
\text { dificultades. } \\
\text { Realizar seguimiento y } \\
\text { detectar posibles problemas. } \\
\text { Orientar en la planificación. }\end{array}$ & $\begin{array}{l}\text { Foro discusión } \\
\text { (seguimiento) } \\
\text { Correo } \\
\text { electrónico } \\
\text { Ficha } \\
\text { seguimiento }\end{array}$ \\
\hline IV & Septiembre & Virtual grupo & $\begin{array}{l}\text { Valorar la adquisición de } \\
\text { competencias. } \\
\text { Reflexionar en relación con } \\
\text { los puntos fuertes y débiles. } \\
\text { Orientar sobre formación } \\
\text { continuada. } \\
\text { Valorar el nivel de estrés } \\
\text { percibido. } \\
\text { Valorar VIRTICS. }\end{array}$ & $\begin{array}{l}\text { Foro discusión } \\
\text { Correo } \\
\text { electrónico } \\
\text { Chat } \\
\text { (seguimiento) } \\
\text { Foro (ficha } \\
\text { seguimiento, } \\
\text { formación } \\
\text { continuada) } \\
\text { Cuestionario } \\
\text { evaluación }\end{array}$ \\
\hline
\end{tabular}

Tabla 1. Resumen del plan de trabajo del tutor 
La modalidad de comunicación será virtual, ocasionalmente habrá encuentros presenciales para realizar la presentación (curso para el personal de enfermería de nueva incorporación), para realizar el grupo de discusión y a petición del profesional novel.

Los recursos utilizados para implantar VIRTICS son:

- Material de apoyo elaborado: material básico, plan de acción tutorial (PAT), guía de uso para los tutorados, guía de uso para el tutor.

- $\quad$ EVT VIRTICS.

- Coordinadora y tutora.

\section{Acciones previas al inicio de VIRTICS}

Las acciones previas llevadas a cabo son:

- Elaboración de un plan de acción tutorial (PAT).

- Reunión con la administradora del espacio: esta persona es personal del laboratorio de aplicaciones telemáticas (LATE) de la URV y persona de apoyo del programa piloto, el objetivo era definir las necesidades de funcionamiento del EVT, sobretodo las adaptaciones a hacer sobre funcionalidades específicas de la tutoría de la URV así como administración de los diferentes cuestionarios que se quería integrar dentro del espacio, con formato web.

- Reunión con la adjunta de formación del HTVC: para presentar el diseño inicial de VIRTICS y concretar el material básico de apoyo que se decide tener disponible como recursos en el espacio, también se acordó y definió el perfil de la tutora abriendo una convocatoria para presentación de candidatas.

- Elección de la tutora virtual, teniendo en cuenta las competencias y habilidades necesarias para dar respuesta a las necesidades y tareas que se le van a plantear. Algunos de estos atributos son: empatía, proacción, ser buen anfitrión, buen comunicador, expertez didáctica (García Aretio, 2001). 
- Reunión con la tutora: entrevista realizada para tener en cuenta el punto de vista de la tutora a partir de su experiencia, una vez presentado el diseño inicial del espacio, siempre abierto a aportaciones y sugerencias.

- Presentación del programa piloto de tutorización virtual VIRTICS a los potenciales participantes dentro del curso para el personal de enfermería de nueva incorporación, organizado por formación continuada del HTVC, durante el mes de junio de 2008. Este curso lo deben realizar todos los profesionales de enfermería antes de iniciar su actividad laboral en el mencionado hospital, y forma parte del programa de acogida. Por lo tanto, se creyó que era el momento y lugar idóneo para presentar conjuntamente los dos programas de tutorización existentes, el que el HTVC ponía a disposición de los profesionales de enfermería debutantes, el programa COM_VATC (presencial), y el programa piloto VIRTICS (virtual). Durante la sesión se visitó el espacio virtual y se explicaron algunas de las funcionalidades y prestaciones que ofrecía, también se comentaron los objetivos y funciones de los tutorados, y se respondió a las dudas que surgieron por parte de las potenciales participantes, y de esta manera se hizo una primera toma de contacto.

- Sesión de trabajo con la administradora del espacio: para concretar aspectos técnicos de funcionamiento se realizaron diferentes pruebas y se diseñó el espacio definitivo.

- Sesión de trabajo con la tutora del espacio: para familiarizarse con el uso del espacio y posibles dudas de funcionamiento.

- Elaboración de las guías de uso: tutor y tutorado, estas están incluidas en el apartado tutorías del EVT.

- Diseño de las diferentes fichas y cuestionarios de evaluación del programa, uno para los tutores y otro para los profesionales tutorados, incluidos dentro del EVT en formato web.

- Alta de los participantes definitivos por parte de la administradora con la previa solicitud de la coordinadora de la autorización por formar parte de la prueba piloto de VIRTICS, DNI, fecha de nacimiento y correo electrónico para formalizar la alta al EVT. 
- Correo electrónico a los diferentes participantes por parte de la administradora para facilitar las claves de acceso e invitarlos a entrar al espacio, dando instrucciones claras de acceso: $<$ http://late-dpedago.urv.cat/moodle/ $>$. Este fue el momento real del inicio de la actividad en el EVT.

\section{Inicio actividad VIRTICS}

El inicio de la actividad de VIRTICS fue el 1 de julio, aunque debido a que los primeros días de contrato laboral las enfermeras noveles formaban parte del programa de tutorización presencial, se observó una baja actividad, durante los primeros días. Este aspecto se debe intentar resolver para próximas ediciones. Una de las acciones propuestas es empezar con una reunión presencial donde presentar el funcionamiento del programa. Además, durante la primera semana de funcionamiento surgió un problema técnico con el servidor que provocó la pérdida parcial de información disponible en el espacio con algunos de los mensajes de participación a los foros iniciales incluidos (presentación y bienvenida). Se tomó la decisión de proponer un grupo de discusión presencial para motivar e implicar a los participantes en el programa, los resultados a partir del grupo de discusión fueron los esperados, ya que a partir de aquel momento el espacio empezó a funcionar.

\section{RESULTADOS}

A continuación se presentan resultados del seguimiento de la implantación del programa piloto de tutorización virtual VIRTICS realizado, con el objetivo de acompañar a los profesionales de enfermería noveles en el proceso de inserción y adaptación al mundo profesional, concretamente al HTVC. Los instrumentos de recogida de datos utilizados fueron: grupo de discusión, ficha web inicial y final, registros de seguimiento diario de la coordinadora y de la tutora, cuestionarios web de evaluación tutorizadas/tutora, análisis de los mensajes de los foros de discusión.

- El EVT solo permitió generar automáticamente informes del registro de actividad por curso académico, debido a que el período de implantación de la prueba piloto fueron los meses de verano: junio-septiembre, estos informes se realizaron manualmente.

- El nivel de participación a la EVT por parte de las enfermeras tutorizadas ha sido escaso, la dinamización por parte de la tutora y de la coordinadora costó mucho. 
- La mayoría están de acuerdo en la afirmación que el espacio VIRTICS ha sido un entorno de fácil uso.

- La totalidad de las tutorizadas: se han sentido cómodas o muy cómodas utilizando el espacio, y están de acuerdo o muy de acuerdo con la afirmación «estoy satisfecho con mi participación en el programa de tutorización», que los programas de tutorización son imprescindibles para la inserción laboral, con las tutorías se mejora la calidad de los cuidados, la duración del programa ha sido adecuada, la calidad del soporte recibido por parte de la tutora, con la afirmación «la tutora me ha hecho sentir bien», con la relación de confianza establecida con la tutora, con la frecuencia de interacción con la tutora que ha sido tan frecuente como se ha necesitado, que la información proporcionada en el espacio le ha sido útil para la inserción profesional.

- El 100\% está de acuerdo que el programa VIRTICS le ha ayudado a afrontar mejor las demandas del mundo profesional.

- El 25\% está muy de acuerdo que «VIRTICS ha influido positivamente en mi motivación», y en que «VIRTICS ha influido en aumentar mi grado de seguridad personal», un 50\% está de acuerdo, el resto está en desacuerdo.

- $\quad$ El 75\% está de acuerdo que «VIRTICS le ha ayudado a aumentar su nivel competencial».

- El 50\% está de acuerdo o muy de acuerdo que «VIRTICS le ha ayudado a disminuir el nivel de estrés producido por la inserción laboral».

- Un 75\% está de acuerdo o muy de acuerdo que la participación en VIRTICS, en general, le ha ayudado a incorporarse al mundo profesional.

- Al solicitar indicar cuales han sido las herramientas del entorno que les han resultado más útiles, el 50\% señalan el foro de discusión y el 50\% restante el correo electrónico.

- Respecto a los retos o dificultades concretas que ha supuesto el programa de tutorización virtual, se indican:

o «He tenido poco tiempo para conectarme» (enfermera 1). 
o «El problema que encuentro es que no sirve para respuestas rápidas, está bien como foro para saber como le va al resto de compañeras y darnos ánimos entre todas, si en algún momento necesitas alguna información que no sea urgente, la puedes solicitar y la tutora virtual te ayuda a buscarla» (enfermera 3).

- No hubo comentarios ni sugerencias de mejora sobre las principales dificultades en el uso de VIRTICS, pues todas coincidieron en la inexistencia de dificultades, ya que consideraban que era un programa sencillo de utilizar.

\section{DISCUSIÓN Y CONCLUSIONES}

En primer lugar, partimos de la base de que la implantación del programa de tutorización VIRTICS respondía a una necesidad detectada de apoyo al proceso de inserción laboral de los profesionales de enfermería noveles. Atendiendo a los resultados obtenidos referentes al funcionamiento del EVT, podemos decir que a pesar de que el espacio ha tenido una actividad relativamente baja y teniendo en cuenta el bajo número de participantes incluidos en este estudio de caso, se valora positivamente, porque ha servido para iniciar un programa de tutoría virtual que ha permitido recoger evidencias en el mismo espacio para su evaluación. La evaluación del EVT ha sido positiva en la mayoría de los aspectos evaluados por parte de todos los miembros de VIRTICS: satisfacción con el uso del programa, soporte del tutor, facilidad de uso del EVT, ayuda para afrontar las demandas del mundo profesional, aumento de motivación y del nivel competencial... Estos resultados coinciden con las valoraciones positivas de otras experiencias piloto de programas de e-tutoría revisados (Pérez Juarez, Verdú et al., 1998; Marabotto y Grau, 1999; Scott, 2005; Vizcaya et al., 2006; Effken, Boyle e Isenberg, 2008; Gisbert, Montserrat y Holgado, 2008; Montserrat, 2010; Lleixà, 2008, 2010, 2011).

Se valora positivamente la implantación del EVT, aunque el nivel de participación ha sido escaso, influido por el reducido número de participantes. Otras experiencias piloto tienen resultados de escasa participación semejante. Gisbert, Montserrat y Holgado (2008), por ejemplo, en un estudio similar recomiendan que uno de los objetivos de la prueba piloto debe ser dinamizar la participación en el programa.

El tiempo de duración de la prueba piloto, tres meses, ha sido considerado adecuado por parte de las participantes de VIRTICS. Existe evidencia internacional que los tres primeros meses de ejercicio profesional son determinantes para adquirir unas habilidades correctas de gestión clínica; se destacan factores que 
favorecen la transición como: programas de orientación formal, programas de acogida, congruencia con la asignación de tareas y/o responsabilidades, programas de tutorización formales por parte de expertos... Estos programas ofrecen apoyo psicosocial y apoyo o instrucción clínica, y aumentan la autoconfianza, la satisfacción con el trabajo realizado; asimismo, estimulan el interés por la práctica clínica (Annand, 1997; Greendwood, 2000; Scott, 2005). Muchas experiencias recomiendan alargar la duración de estos programas entre seis y doce meses, ya que consideran que es el tiempo de adaptación y/o transición necesario (Ebright, Urden et al., 2004; McCloughen y O'Brien, 2005; Forman, 2006; Donner y Wheeler, 2007). En este sentido, hay que valorar este aspecto en el futuro.

Algunos de los problemas de acceso detectados tanto a nivel individual manifestados por algunas de las participantes, como problemas del mismo servidor, provocan un alto nivel de frustración entre las participantes. Hay que destacar la importancia de la continuidad de este proyecto para dar respuesta a la necesidad de apoyo y soporte emocional detectada en los profesionales de enfermería noveles. Pensamos que no se puede detener, y que se debe continuar trabajando para mejorar el EVT y el programa VIRTICS en general. Y es que, como señala Prats (2007, p. 68), "todo proyecto de innovación con el apoyo TIC pide cuatro virtudes: confianza, tiempo, paciencia y perseverancia, partiendo siempre de un plan apoyado por la institución". Para ello, ahora se inicia un proceso reflexivo para una vez descrita la experiencia, volvamos la mirada hacia aquellos aspectos que pueden ser visiblemente mejorables en próximas ocasiones. De su revelación solo cabe esperar una postura autocrítica, y como consecuencia de esta, la intención de compartir experiencias que ayuden a salvar los errores por los que nosotros ya hemos pasado.

Por último, debemos continuar investigando sobre cuestiones y problemas que afectan directamente a la profesión, intentando dar respuesta a las necesidades detectadas, contribuyendo y ayudando a la evolución y mejora de la inserción laboral. Como conclusión final, podemos destacar que, si un programa de e-tutoría se utiliza para enriquecer personalmente y profesionalmente a una persona, así como para maximizar su potencial, se trata sin duda de un programa muy válido en enfermería, así como en otras profesiones, con grandes potencialidades y que puede no tener límite (Donner y Wheeler, 2007). 


\section{REFERENCIAS BIBLIOGRÁFICAS}

Annand, F. (1997). The mentor commitment. Insigth, 22 (2), (41-45).

Donner, G. J.; Wheeler, M. M. (2007). A guide to coaching and mentoring in nursing. Trends and issues in nursing 21. International Council of Nurses cop. Ebright, P. R.; Urden, L. et al. (2004). Themes Surrounding novice nurse nearmiss and adverse-event situations. The Journal of nursing administration, 34 (11), (531-538).

Effken, J. A.; Boyle, J. S.; Isenberg, M. A. (2008). Creating a virtual research community: the University of Arizona $\mathrm{PhD}$ program. Journal of professional nursing, 24 (4), (246-253).

Ezeiza, A. (2007). Tutoría on-line en el entorno universitario. Comunicar, 29, (149-156).

Forman, C. (2006). The orientation of nurses in New Work settings. Geneva: International Council of Nurses.

García Aretio, L. (2001). La educación a distancia. De la teoría a la práctica. Barcelona: Ariel.

Gisbert, M.; Montserrat, S.; Holgado, J. (2008). Experiencia piloto de implantación del plan de acción tutorial en el Máster en Tecnología Educativa: E-learning de la Universidad Rovira y Virgili. La acción tutorial en la universidad del siglo XXI. Sevilla, RED-U, Universidad Pablo Olavide y Universidad de Sevilla.

Greendwood, J. (2000). Critique of the graduate nurse: an international perspective. Nurse Education Today, 20 (1), (17-23).

Lleixá, M. (2008). La tutoria virtual i la inserció laboral dels professionals dinfermeria. Tesis doctoral. Departamento de Pedagogía, Universitat Rovira i Virgili.

Lleixá, M.; Albacar, N.; Montesó, P.; Ferré C.; Dalmau, P.; Adell, B.; Berenguer,
M.; Marqués, L.; Montserrat, S. (2010). La tutòria virtual en el pràcticum: està satisfet l'alumnat? Revista Àgora d'Infermeria, 14 (56), (149-55).

Lleixá, M.; Gisbert, M.; Marqués, L.; Espuny, C. (2011). Diseño de un programa de e-tutoría para favorecer la inserción laboral de los profesionales noveles de enfermería. Revista Pixel Bit. Revista de medios y educación, 38, (23-34).

Marabotto, M. I.; Grau J. E. (1999). La tutoría telemática en la educación a distancia. RIED. Revista Iberoamericana de Educación a distancia, 2 (1), (117-38).

McCloughen, A.; O'brien, L. (2005). Development of a mentorship programme for new graduate nurses in mental health. International journal of mental health nursing, 14 (4), (276-84).

Montserrat Pera, S.; Gisbert Cervera, M.; et al. (2005). e-Tutor: Towards a tool to facilitate the development of academic support processes at the university level. Third International Conference on Multimedia Information and Comunication Tecnologies in Education. Cáceres (Spain). [en línea] Disponible en: www.formatex.org/ micte2005/120.pdf (consulta 2010, 13 de septiembre)

Montserrat, S.; Gisbert, M.; Isús, S. (2007). E-tutoria: uso de las tecnologías de la información y de la comunicación para la tutoría académica universitaria. Revista Electrónica Teoría de la Educación. Educación y Cultura en la Sociedad de la Información, 8 (2). [en línea] Disponible en: www. usal.es/ teoriaeducacion/rev numero $08 \quad 02 / \mathrm{n} 8 \quad 02$ sogues gisbert isus.pdf (consulta 2010, $18 \mathrm{de}$ agosto)

Montserrat, S. (2010). La tutoria en els processos d'ensenyament aprenentatge desenvolupats a través 
d'entorns virtuals d'aprenentatge. Tesis Doctoral. Departamento de Pedagogía, Facultad de Ciencias de la Educación y Psicología. Universidad Rovira y Virgili. Tarragona.

Pallof, R. M.; Pratt, K. (1999). Building learning communities in cyberspace. Effective strategies for the "on line" classroom. San Francisco: Jossey-Bass.

Pavón, F.; Casanova, J. (2007). Experiencias docentes apoyadas en aulas virtuales. RIED. Revista Iberoamericana de Educación a distancia, 10 (2), (149-63). Pérez Juárez, M. A.; Verdú, M. J.; Rodríguez Pajares, B.; Mompó R.; Navazo M. A.; López Fernández, R.; García Carrasco, J. (1998). Diseño de un instrumento de aprendizaje para la formación continúa de trabajadores de PYMES. RIED. Revista Iberoamericana de Educación a distancia, 1 (2), (119-41).

Prats, M. A. (2007). De l'escola analògica a l'escola digital: noves formes d'aprendre. En: Riera J.; Roca E. Coordinadors. Reflexions sobre educació en una societat corresponsable. Collecció
Antines: 13. Barcelona: Cossetània Edicions.

Rísquez, A. (2006). E-mentoría: avanzando la investigación construyendo la disciplina. Revista Complutense de Educación, 17 (2), (121-35).

Sedeño, A. M. (2006). Tutoría universitaria y asignaturas de medios audiovisuales. Comunicar, 27, (211-217).

Scott, E. S. (2005). Peer-to-peer mentoring. Teaching collegiality. Nurse educator, 30 (2), (52-66).

Rodríguez Santero, J.; Valverde, A. (2003). Tecnologías al servicio de la orientación y de apoyo al aprendizaje. Comunicar, 20, (89-95).

Vizcaya, M. F.; Pérez Cañaveras, R. M.; et al. (2006). Experiencia de Acción Tutorial Virtual en la Docencia Práctica Clínica en los Estudios de Enfermería. IV Jornades de Xarxes d'Investigació en Docència Universitària. Alicante: Universitat d'Alacant. [en línea] Disponible en: www.eduonline.ua.es/ web ice/comunicaciones/3P2.pdf (consulta 2011, 2 de octubre).

\section{PERFIL ACADÉMICO Y PROFESIONAL DE LOS AUTORES}

Mar Lleixà Fortuño. Doctora por la Universidad Rovira i Virgili (URV), diplomada en enfermería Universidad de Barcelona, licenciada en Psicología UNED, Máster en Tecnología Educativa: e-learning y gestión del conocimiento URV, profesora del Departamento de Enfermería de la URV Campus Terres de l'Ebre, coordinadora del Máster en Ciencias de la Enfermería de la URV, miembro del grupo de investigación de enfermería de la URV CARING.

E-mail: mar.lleixa@urv.cat

Cinta Espuny Vidal. Doctora por la URV, Maestra, especialista universitaria en Tecnologia Educativa: Diseño de Materiales Multimedia para la Educación. Máster en Tecnologia Educativa: e-learning y gestión del conocimiento, profesora del Departamento de Pedagogía de la URV Campus Terres de l'Ebre. Miembro del Grupo de Investigación ARGET (Applied Research Group in Education and Technology, de la URV, 2009 SGR 596), e investigador del proyecto SIMUL@ Evaluación 
de un entorno tecnológico de simulación para el aprendizaje de competencias transversales en la universidad (EDU2008-01479).

E-mail: cinta.espuny@urv.cat

Juan González Martínez. Doctor en Filología Hispánica. Ldo. en Antropología Social y Cultural. Especialista en Docencia en EEES. Profesor funcionario de instituto. Profesor de la Universitat Rovira i Virgili, Campus Terres de l'Ebre, Departamento de Pedagogía. Miembro del Grupo de Investigación ARGET (Applied Research Group in Education and Technology, de la URV, 2009 SGR 596), e investigador del proyecto SIMUL@Evaluación de un entorno tecnológico de simulación para el aprendizaje de competencias transversales en la universidad (EDU2008-01479).

E-mail: juan.gonzalezm@urv.cat

Mercè Gisbert-Cervera. Doctora en Ciencias de la Educación por la UB. Profesora Titular de Universidad Departamento de Pedagogía de la U. Rovira i Virgili de Tarragona. Codirectora del Màster Oficial: Tecnología Educativa: e-Learning y gestión del conocimiento y coordinadora del doctorado en Tecnología Educativa. Directora del Grupo de Investigación ARGET (Applied Research Group in Education and Technology, de la URV, 2009 SGR 596), e investigadora principal del proyecto SIMUL@Evaluación de un entorno tecnológico de simulación para el aprendizaje de competencias transversales en la universidad (EDU2008-01479).

E-mail: merce.gisbert@urv.cat

Luis Marqués-Molías. Doctor en Ciencias de la Actividad Física y el Deporte por la Universidad de Zaragoza y Máster en Tecnología Educativa: e-learning y gestión del conocimiento por la Universidad Rovira i Virgili. Actualmente coordinador académico del Máster en Tecnología Educativa: e-learning y gestión del conocimiento de la URV y profesor del Departamento de Pedagogía. Miembro del Grupo de Investigación ARGET (Applied Research Group in Education and Technology, de la URV, 2009 SGR 596). Ha participado en diversos proyectos de investigación en el ámbito de la tecnología educativa y autor de varias publicaciones en la materia.

E-mail: luis.marques@urv.cat

Nuria Albacar Riobóo. Diplomada en enfermería, Máster en Ciencias de la Enfermería URV, profesora del Departamento de Enfermería de la URV, miembro del grupo de investigación de enfermería de la URV CARING.

E-mail: nuria.albacar@urv.cat 
DIRECCIÓN DE LOS AUTORES

\author{
Universitat Rovira i Virgili \\ Campus Terres de l'Ebre \\ Avenida Remolins 13-15, \\ 43500 Tortosa, Tarragona, España
}

Fecha de recepción del artículo: 21/12/2011

Fecha de aceptación del artículo: 10/4/2012

\title{
Como citar este artículo:
}

Lleixà Fortuño, M.; Gisbert-Cervera, M.; Marqués-Molías, L.; Espuny Vidal, C.; González Martínez, J.; Albacar Riobóo, N. (2013). Tutoría virtual para profesionales de enfermería noveles. RIED. Revista Iberoamericana de Educación a Distancia, volumen $16, \mathrm{n}^{0} 1$, pp. 199-215. 